\title{
Why Don't Medical Practitioners Treat Malaria Rationally? A Qualitative Study from Pakistan
}

\author{
Madeeha Malik*, Mohamed Azmi Ahmad Hassali, Asrul Akmal Shafie and \\ Azhar Hussain \\ Discipline of Social and Administrative Pharmacy, School of Pharmaceutical Sciences, Universiti Sains Malaysia, \\ 11800 Minden, Penang, Malaysia.
}

\begin{abstract}
Purpose: To explore medical practitioners' perceptions towards irrational malaria treatment practices in Pakistan.

Methods: A qualitative study was designed to explore the perceptions of medical practitioners regarding antimalarial prescribing practices in two major cities of Pakistan, namely, Islamabad (national capital) and its twin city, Rawalpindi. Semi-structured interviews were conducted using in-depth interview guides to collect data. Nineteen interviews with doctors working at different public and private hospitals in Islamabad and Rawalpindi were conducted at a place and time convenient for the respondents. The interviews were audio-taped, transcribed verbatim, and evaluated by thematic content analysis and other author analysis.

Results: The interviews focused on three major components, i.e., treatment practices in malaria and influencing factors, role of Malaria Control Program, and suggestions for improvements. Thematic content analysis of these components yielded further themes: (1) Prevalence of malaria, (2) Common trends of treatment, (3) Current scenario of rational drug use, (4) Major contributing factors to irrational drug use, (5) Use of antibiotics, (6) Role of healthcare system, (7) Role of Malaria Control Program, (8) Role of hospital pharmacist, (9) Collaborative efforts of doctors and pharmacists in promoting rational treatment practices, and (10) Strategies to improve current treatment practices.

Conclusion: The current study showed that all the respondents in the two cities agreed that irrational prescribing practices, unavailability of drugs, lack of awareness and adherence of prescribers to standard treatment guidelines, are the major factors contributing to irrational drug use in malaria in Pakistan.
\end{abstract}

Keywords: Health practitioners, Malaria, Qualitative methodology, Rational drug use, Treatment practices. 


\section{INTRODUCTION}

Malaria continues to be a major public health issue in Pakistan due to socio-economic and epidemiologic reasons [1]. Development of resistance to antibiotics, ineffective treatment, adverse effects, drug dependence and economic burden to the patient and society are the major obstacles hindering effective malaria therapy [2]. Enormous resources are reported to be wasted when drugs are prescribed, dispensed, administered and used irrationally [3].

Malaria is conventionally diagnosed through clinical impression, and most presenting fever cases are suspected and treated for malaria without evidence of malaria parasitaemia. This practice expose patients to undue drugrelated problems including side effects, treatment costs, and also raises concerns regarding increasing resistance and overestimation of the burden of the disease [4]. When symptoms appear, patients often self-treat and later seek advice from formal healthcare providers working in public and/or private healthcare facilities. Studies carried out in several developing countries including Pakistan have shown major prescribing problems due to polypharmacy, overuse of antibiotics and injections, wrong drugs, unsuitable doses, dosages and improper duration of treatment of antimalarial drugs [57]. Most of the medical practitioners in these countries are inclined to adopt their own protocols to treat malaria rather than adhering to standard regimens [8-9].

There are many problems associated with current treatment practices for malaria in the country. This calls for an in-depth investigation of the factors and motivations that underlie problems in prescribing practices in order to treat malaria in a more rational way. Therefore, the main objective of the present study was to investigate health practitioners' perceptions towards factors underlying irrational malaria treatment practices in two Pakistani cities - Islamabad (national capital) and Rawalpindi (its twin City).

\section{EXPERIMENTAL}

\section{Study design}

A qualitative study was designed to explore the perceptions of doctors regarding rational use of drugs in the treatment of malaria, factors responsible for irrational drug use, role of the healthcare system and malaria control program in promoting rational drug use, and strategies to improve drug use and control of malaria. The study protocol was approved by the Malaria Control Program, Ministry of Health, Pakistan. Semi-structured interviews were conducted using in-depth interview guides to collect the data.

\section{Participants}

The study participants were medical practitioners working in tertiary and secondary healthcare facilities and as general practitioners. The sampling frame comprised of professionally qualified medical practitioners working in both public and private tertiary and secondary healthcare facilities and also practicing as general practitioners in their private clinics in the evening in two Pakistani cities namely, Islamabad (national capital) and its twin city of Rawalpindi.

\section{Sample size and sampling technique}

Nineteen medical practitioners took part in the study. Non-probability sampling technique, i.e., snowball sampling, was adopted. Snowball sampling is the best way to identify respondents with certain attributes or characteristics and is influential in difficult to reach populations [10-11]. In snowball sampling, the researcher identifies the first respondent, and then the respondent is asked to suggest more research participants. 


\section{Study tool}

A semi-structured interview guide was developed after extensive literature review and used as the study tool. A number of aspects to be addressed were already identified from the literature which ensured the inclusion of key issues regarding rational drug use in the treatment of malaria. While designing the questions, the focus was to keep them as open as possible to interviewees for maximum opportunity to express their views. The first draft of the interview guide was discussed among the authors and was modified after a few rounds of discussion. Pre-testing of the interview guide was carried out with nine doctors to check whether particular questions were useful in the retrieval of information. Specific probes were identified during the pilot interviews and the interview guide was subsequently modified. Semi-structured oneto-one interviews were conducted since it is the most practical and convenient way for busy professionals and thematic saturation was considered to be a cut-off point to stop the sampling of subjects [12].

\section{Procedure and interview process}

All the interviews were conducted from April to May 2011 at the offices of the respondents. Nineteen respondents, via snowball sampling technique, were recruited from tertiary secondary healthcare facilities and private clinics healthcare facilities in Islamabad and Rawalpindi, Pakistan. Data collection was stopped at a point when no new themes emerged [12]. The identified participants were contacted in person or on phone to fix interview appointments. Written consent was obtained from each of the participants prior to the conduction of interview. Probing questions were used where necessary and participants were given freedom to express their views at the end of the interview session. Each interview lasted approximately 20 - $30 \mathrm{~min}$. All the interviews were conducted in the local language and at times convenient for the respondents.
Permission for recording was obtained and all the interviews were recorded. Each interview was transcribed verbatim. Transcribed interviews were subjected to thematic content analysis, and the transcripts were analyzed for relevant content to identify emerging categories [12].

\section{RESULTS}

Most of the respondents $68.4 \%(\mathrm{n}=$ 13), were male and had Bachelor of Medicine, Bachelor of Surgery (MBBS) qualification. Most of the respondents $52.7 \%(n=10)$ were working in private tertiary healthcare facilities along with practice in private clinics while $47.3 \%(n=9)$ were employed in public tertiary healthcare facilities. Detailed demographics of the respondents are shown in Table 1.

\section{Theme 1: Prevalence of malaria in Pakistan}

Malaria was identified as a seasonal disease that is mostly prevalently from August to November. Plasmodium vivax is the common type of malaria observed in Punjab, as stated by all the doctors.

"Malaria is a seasonal disease and most of the patients with fever are seen from August till November but not necessarily confirmed patients of malaria. P. Vivax is the common type of malaria in Punjab" (Doc.3).

"I have come across many patients with fever during the season of malaria but usually malaria is not confirmed in our country. $P$. Vivax is usually the common type of malaria in Punjab" (Doc 7).

\section{Theme 2: Common trends of treatment for malaria}

Chloroquine, sulphadoxine/pyremethamine (Fansidar) or artemether/lumefentrine combinations were seen as the most commonly prescribed drugs for the treatment of malaria by the doctors. 
Table 1: Demographic characteristics of the medical practitioners

\begin{tabular}{|c|c|c|c|c|c|}
\hline Code & Gender & Qualification & Experience & Sector & Current designation \\
\hline Doc 1 & Male & MBBS/FCPS & 18 years & Public & Head of medicine department/ G.P \\
\hline Doc 2 & Female & MBBS/MPH & 6 years & Public & Medical officer/ G.P \\
\hline Doc 3 & Female & MBBS & 2 years & Private & House officer/ G.P \\
\hline Doc 4 & Male & MBBS & 1 year & Private & House officer/ G.P \\
\hline Doc 5 & Male & MBBS & 2 years & Public & House officer/ G.P \\
\hline Doc 6 & Male & MBBS & 4 years & Private & House officer/ G.P \\
\hline Doc 7 & Male & MBBS & 2 years & Private & House officer/ G.P \\
\hline Doc 8 & Male & MBBS & 3 years & Public & House officer/ G.P \\
\hline Doc 9 & Female & MBBS/FCPS & 10 years & Private & Head of medicine department/ G.P \\
\hline Doc 10 & Female & MBBS/MPH & 8 years & Private & Medical officer/ G.P \\
\hline Doc 11 & Male & MBBS/MPH & 7 years & Public & Medical officer/ G.P \\
\hline Doc 12 & Male & MBBS/MPH & 8 years & Private & Medical officer/ G.P \\
\hline Doc 13 & Male & MBBS/MPH & 9 years & Private & Medical officer/ G.P \\
\hline Doc 14 & Male & MBBS/FCPS & 20 years & Public & Consultant/ G.P \\
\hline Doc 15 & Male & MBBS/FCPS & 17 years & Private & Consultant/ G.P \\
\hline Doc 16 & Female & MBBS & 1 year & Private & House officer/ G.P \\
\hline Doc 17 & Female & MBBS & 2 years & Public & House officer/ G.P \\
\hline Doc 18 & Male & MBBS/MPH & 7 years & Public & Medical officer/ G.P \\
\hline Doc 19 & Male & MBBS/MPH & 8 years & Public & Medical officer/ G.P \\
\hline
\end{tabular}

"Chloroquine, fansidar or artemether/ lumefentrine combinations are usually prescribed in treating malaria" (Doc 13).

"Most of the patients do respond to chloroquine as first line treatment while Fansidar is prescribed as second line agent and artemether/lumefentrine combination is given when patients do not respond to chloroquine and/or Fansidar" (Doc 9).

\section{Theme 3: Current scenario of rational drug use in treatment of malaria}

All the respondents agreed that malaria is treated irrationally in the healthcare facilities and inappropriate diagnosis and treatment on the basis of empirical therapy were considered as the factors responsible for such practices.

"Malaria is mostly treated on the basis of empirical therapy in the country due to patient's unaffordability for diagnostic test which might lead to overtreatment of malaria and this is promoting irrational drug use" (Doc.1).
"Patients are reluctant to go for diagnostic tests because of this malaria is usually treated on the basis of empirical therapy. Inadequate lab support and inappropriate diagnostic procedures are the most important current concerns to be addressed for irrational drug use in malaria in the country" (Doc 5).

\section{Theme 4: Major contributing factors in irrational drug use in the treatment of malaria}

In view of most of the doctor's inappropriate diagnosis, irrational prescribing practices, unavailability of drugs, lack of awareness of prescribers regarding standard treatment guidelines and self medication are the major factors promoting irrational drug use in the treatment of malaria in Pakistan.

"Inappropriate diagnosis, lack of adequate lab support and medical practice by a large number of quacks are the most prominent factors promoting irrational drug use in Pakistan" (Doc. 12). 
"Inappropriate diagnosis, peer pressure, unavailability of drugs and self-medication are the biggest hurdles in rational treatment of malaria" (Doc.4).

"I think poly pharmacy and lack of awareness of prescribers regarding standard treatment guidelines are promoting irrational prescribing practices and drug use" (Doc.1).

\section{Theme 5: Use of antibiotics and injections in treatment of malaria}

Although irrational prescribing of antibiotics is a common practice but irrational use of injections is not frequent while treating malaria, as stated by most of the doctors.

a) Irrational prescribing of antibiotics in the treatment of malaria

"I think that over prescribing of antibiotics in malaria is not only promoting irrational drug use but also promoting resistance and we are losing effective medicines such as quinolones" (Doc.8).

"Yes, antibiotics are being prescribed irrationally as usually malaria parasite is not confirmed through lab diagnostic test and antibiotics are prescribed in anticipation of diagnosis of malaria or typhoid. This overuse is leading to anti malarial drugs resistance" (Doc.3).

"Yes, they are being prescribed irrationally as malaria is mostly treated empirically and symptoms are often confused with those of typhoid. Peer influence is another factor in promoting over use of antibiotics in case of malaria" (Doc15).

b) Irrational prescribing of injections in the treatment of malaria

"I don't think irrational use of injection is common in case of malaria" (Doc.4).

"I don't see any over use of injections in treating malaria in the urban areas but it might not be true for the practices in rural areas" (Doc.2).

\section{Theme 6: Role of health care system in rational treatment practices in malaria}

Almost all the respondents were of the view that the health care system has failed to play a positive role in promoting rational treatment of malaria in Pakistan; even then public sector is comparatively more rational in their practices than the private sector.

"I think private sector is an emerging sector at the present time and I agree that they have more facilities and are economically sound. But I think the public sector which is regulated by government and has more experienced practitioners, is better in practice than the private sector" (Doc.1).

"I think public sector is better due to provision of better diagnostic facilities and free of cost of treatment which in turn increases patient compliance" (Doc14).

"I think public sector practitioners are more rational in practice as prescribing of drugs is according to the policies of ministry of health and prescriber's choice is not much involved in the treatment" (Doc. 8).

\section{Theme 7: Role of Malaria Control Program (MCP)}

All the doctors agreed that Malaria Control Program of the Ministry of Health has failed to play its role effectively in promoting rational drug use and control of malaria in Pakistan.

"I think Malaria control program has failed to control malaria and promote rational drug use in malaria treatment in the country" (Doc.17).

"No, the Malaria control program is not playing any effective role at all. I see the program highly inefficient" (Doc.6).

"Malaria control program is there but is not effective. I think concerned professional 
doctors should be involved in the program for enhancing the impact of the program on the disease as well as on the community" (Doc. 19).

\section{Theme 8: Role of hospital pharmacist in promoting rational drug use}

All the respondents agreed that the hospital pharmacist has an immense role to play in promoting rational drug use but have not come across any pharmacist playing a clinical role in hospitals in Pakistan.

"I think the role of hospital pharmacist in the west is immense but I have not come across any clinical role of pharmacist in the hospitals in our country" (Doc.3).

"Although pharmacists have been appointed in hospitals, but I don't see any clinical role performed by them, usually I see their role being performed by unqualified dispensers in our health care system" (Doc. 18)

"I think if the pharmacist performs appropriate drug selection, procurement and dispensing he is the best person to promote rational drug use in the health care system. But unfortunately he is not performing any effective clinical role except being a store keeper" (Doc.9).

Theme 9: Collaborative efforts of doctors and pharmacists in promoting rational treatment practices

Most of the respondents were of the view that collaboration of doctors and pharmacists can promote rational drug use in the treatment of malaria.

"Yes, it can help to reduce adverse drug reactions and endorse more effective patient counseling" (Doc. 11).

"I think definitely team work can promote better services as patient counseling is usually neglected due to immense workload" (Doc.7).
Theme 10: Strategies to improve current treatment practices for control of malaria

In the view of most of the respondents, improved diagnostic and treatment facilities, prescribing by generic names, training of health professionals, implementation of standard treatment guidelines for malaria in the healthcare system and more effective role of Malaria control program are the most effective strategies to promote rational drug use and control of malaria in Pakistan.

\section{a) Improved diagnostic and treatment facilities}

"I think improved diagnostic facilities and counseling of patients can help to improve the current situation" (Doc.9).

"Improved diagnostic facilities and drug supply at the facilities and more preventive measures can help in promoting rational drug use and control of malaria" (Doc1).

\section{b) Prescribing by generic names}

"Yes. I assume generic prescribing can be a tool in promoting rational drug use" (Doc.2).

"I think generic prescribing will be effective in promoting rational drug use if implemented in the country" (Doc.7).

"I think generic prescribing cannot be implemented in the country but I would suggest that the number of registration for a particular drug/salt should be limited by the Ministry of health" (Doc.4).

\section{c) Training of health professionals}

"I think by improving education and training of health professionals we will be able to improve the quality of services provided to the patients" (Doc.11).

"The only solution to promote rational drug use is by training of doctors regarding standard treatment guidelines" (Doc.7). 
"Proper training of both doctors and pharmacists regarding standard treatment guidelines should be conducted for promoting rational drug use" (Doc. 19).

\section{d) Implementation of standard treatment guidelines for malaria in the healthcare system}

"I think standard treatment guidelines for malaria must be implemented and strictly followed as this is the ultimate solution to irrational drug use in malaria" (Doc.5).

"Standard treatment guidelines for malaria are available in the country but they are not being followed. But I don't see any involvement of stakeholders at government level in their implementation. They should be implemented in the healthcare system to improve the current situation" (Doc. 8).

\section{e) Effective role of Malaria Control Program}

"Malaria control program must play effective role in promoting awareness regarding malaria control in the country" (Doc.3).

"Malaria control program needs to play more effective role in promoting rational drug use and control of malaria" (Doc.11).

\section{DISCUSSION}

Pakistan is among 109 countries with endemic malaria with an estimated number of 1.5 million annual malaria episodes. It is a major health problem, threatening the health of the people due to prevailing socioeconomic conditions and epidemiological situation. The respondents revealed that malaria is a seasonal disease which is usually prevalent from August to November. Plasmodium.vivax is the common type of malaria being observed in Punjab and chloroquine, sulphadoxine/pyremethamine (fansidar) or artemether/lumefentrine combinations are the most frequently prescribed drugs for the treatment of malaria in Pakistan. Similar patterns of treatments for malaria have been reported by other studies [13].

The respondents agreed that malaria is treated irrationally in the healthcare facilities and inappropriate diagnosis and treatment on the basis of empirical therapy were considered as the factors responsible for such practices. This might be due to patient's inability to afford malaria parasite diagnostic test which is quite expensive. The results of the present study are in line with the study conducted in Nigeria where the use of presumptive malaria diagnosis without laboratory support, was a common diagnostic procedure for malaria and inclined to poor quality of malaria diagnosis and treatment [14]. However, it was observed that prescribing of anti-malarial drugs after laboratory confirmation only decreased the total number of malaria prescriptions significantly in Malawi [15].

Irrational use of drugs in the health care system remains a major challenge to be addressed. The problem of irrational practices in treatment of malaria in health care system, are contributing to the development of resistance, ineffective treatment and economic burden to the patient and society. Irrational prescribing practices, unavailability of drugs, lack of awareness and adherence of prescribers regarding standard treatment guidelines, self medication and overuse of antibiotics were highlighted as the major factors contributing towards irrational drug use in malaria in Pakistan by the respondents. Similar patterns of irrational drug use in other countries have been reported [16-17].

The health care system in Pakistan has not been able to play an effective role in promoting rational drug use in the treatment of malaria. However, the public sector is comparatively more rational in their practices than the private sector. It is often observed that the prescribing and dispensing practices are not up to standard in public and private 
sector health facilities in developing countries, including Pakistan. All the respondents agreed that Malaria Control Program (MCP) has not played its role effectively in promoting rational drug use and control of malaria in Pakistan. This might be due to the fact that MCP has targeted mainly 19 districts in different provinces due to shortage of funds, and has not been able to target tertiary and secondary healthcare facilities. However, national MCPs have been successful in reducing the number of malaria cases in Iran and Uganda [18].

The concept of pharmaceutical care has transformed the pharmacy profession to a more patient-oriented practice which ensures the desired therapeutic outcomes. Pharmacists are a vital source of drug knowledge in clinics, hospitals, and community pharmacies across the world. The role of the pharmacist in patient medication counseling and in providing medication advice to physicians is well acknowledged by the healthcare practitioners in developed countries [19]. Although, the hospital pharmacist has an immense role in promoting rational drug use, all the respondents agreed that they have not come across any pharmacist playing significant clinical role in hospitals in Pakistan. They opined that their role is limited to only dispensing, procurement and inventory control. This might be due to lack of training, orientation and recognition of the pharmacist as a healthcare professional in the Pakistani healthcare system. This situation is similar to what obtains in other developing countries [20].

Most of the respondents were of the view that improved diagnostic and treatment facilities, prescribing by generic names, training of health professionals, implementation of standard treatment guidelines for malaria in the healthcare system and the more effective involvement of MCP are the most effective strategies to promote rational drug use and control of malaria in Pakistan. Various intervention studies have reported significant impact on diagnostic and treatment practices [21-22] .

\section{Limitation of the study}

The study was conducted in two cities of Pakistan. It is most likely that the health practitioners in other parts of the country would have similar perceptions regarding treatment practices for malaria, but the findings may not be generalizable to other cities of the country.

\section{CONCLUSION}

Based on the results of this qualitative study, all the prescribers agreed that irrational prescribing practices, unavailability of drugs, lack of awareness and adherence of prescribers to standard treatment guidelines, self-medication and over-use of antibiotics are the major factors contributing to irrational drug use in malaria in Pakistan. This calls for an in-depth investigation of the healthcare system, factors that motivate irrational practices and the development of appropriate interventions that target these causative factors. The results of this study emphasized that it might be tedious and difficult to change current practices; however, active involvement of Malaria Control Program, different stakeholders and implementation of standard treatment guidelines are required to achieve rational drug use and control of the disease in Pakistan.

\section{REFERENCES}

1. Malik M, Hassali MAA, Hussain A. Strategic solution to malaria eradication in Paki stan. Journal of Pharmacy Practice and Research, 2011; 41(1): 73-74.

2. Juncosa B. Antibiotic Resistance:Blame it on Lifesaving Malaria Drug? 2008 [cited 2010 20th November]. http://www.scientificameri can.com/article.cfm?id=antibiotic-resistanceblame-it-on-lifesaving-malaria-drug

3. Quick J, Laing R, R.-DD. Intervention research to promote clinically effective and economically efficient use of pharmaceuticals. J Clini Epidemiol, 1991; 44(2): 57-65.

4. UNICEF. Promoting Rational Use of Drugs and Correct Case Management in Basic Health 
Services. 2000 [cited 2010 Dec 11]; Available from: $\quad$ http://www.unicef.org/prescriber/eng_ p18.pdf.

5. Chandler Cl, Jones C, Boniface G, Juma K, Reyburn $H$, Whitty CJM. Guidelines and mindlines: why do clinical staff over-diagnose malaria in Tanzania? A qualitative study. Malaria Journal, 2008: 7: 53.

6. C Chareonkul, VL Khun, C Boonshuyar. Rational drug use in Combodia:Study of three pilot health centers in Kampong Thom Province. Southeast Asian J Trop Med Public Health, 2002; 2(33): 418-424

7. Nizamani A, Kalar N, Khush I. Burden of malaria in Sindh, Pakistan: a two years surveillance report. JLUMHS, 2006: 76-83.

8. WHO. Intercountry workshop on monitoring therapeuitc efficacy of anti malarial drugs. 2002 [cited 201022 April]; Available from: www.emro.who.int/rbm/news-yem02.htm

9. Mannan AA,, Malik EM, Ali KM. Antimalarial prescribing and dispensing practices in health centres of Khartoum state. Eastern Mediter Health J, 2009; 15(1): 122-128

10. Bloch A, Methodological challenges for national and multi-sited comparative survey research. J Refug Stud, 2007; 20: 230-247.

11. Jamshed S, Hassali MAA, Ibrahim MIM, Shafie AA, Babar A. Knowledge, Perception and Attitude of Community Pharmacists towards Generic Medicines in Karachi, Pakistan: A Qualitative Insight Trop J Pharm Res 2010; 9(4): 409-415-

12. Hassali MA, Kong D, Stewart K. Generic medicines: perceptions of consumers in Melbourne, Australia. Int J Pharm Pract, 2005; 13: 257264.

13. Malaria Control Program. NMCP in Bangladesh [cited 2011 9th july]. Available from http://www.nmcp.info/
14. Onwujekwe O, Uzochukwu B, Dike N, Uguru N, Nwobi E, Shu E. Malaria treatment perceptions,practices and influences on proider behaviour: comparing hospitals and non-hospitals in south-east Nigeria. Malaria Journal, 2009; 8(246).

15. Chitaka R, Khare AK, Brickling C. Prescribing policy for antimalarials. . African Health 1998; 20(2): 2-5.

16. Chareonkul C, Khun VL, Boonshuyar C. Rational drug use in Cambodia:Study of three plot health centers in Kampong Thom Province. Southeast Asian J Trop Med Public Health, 2002; 2(33): 418-424.

17. Abuakua BK, Korama KA, Binkab FN. Antimalarial Prescribing Practices: A Challenge to Malaria Control in Ghana. Medical Principles Pract, 2005; 14(5): 332-337

18. Raeisi, A., et al., The trend of Malaria in I.R. Iran from 2002 to 2007 Hakim Res J 2009; 12(1): 35-41.

19. Ranelli PL, Biss J. Physicians' Perceptions of Communication with and Responsibilities of Pharmacists Journal of the American Pharmacists Association, 2000; 40(5): 625630.

20. Shaikh, S. Development of hospital pharmacy in Bangladesh. 2009 [cited 201019 Jan ]; Available from: http://www.pjonline.com.

21. Brugha R,,Zwi A. Improving the quality of private sector delivery of public health services: challenges and strategies. Health Policy Planning, 1998; 13(2): 107-120.

22. Nsimba SED, Assessing the impact of educational intervention for improving management of malaria and other childhood illnesses in kibaha district-tanzania. East African Journal of Public Health, April 2007; 4(2): 5-11 